\title{
Stabilization Laws for Collective Motion in Three Dimensions
}

\author{
Luca Scardovi, Rodolphe Sepulchre, and Naomi Ehrich Leonard
}

\begin{abstract}
This paper proposes a framework for the design of control laws that stabilize relative equilibria in a model of identical, steered particles moving in three-dimensional Euclidean space. Under the assumption of all-to-all communication, the derived control laws only require relative orientations and positions. We extend the obtained results in the presence of limited communication topologies by equipping each agent with a consensus estimator.
\end{abstract}

\section{INTRODUCTION}

In recent years particular attention has been devoted to the design of control laws for the coordination of a group of autonomous systems. Applications include sensor networks, where a group of autonomous agents has to collect information about a process by choosing maximally informative samples [1], [2], and formation control of autonomous vehicles (e.g. unmanned aerial vehicles) [3], [5]. In these contexts it is relevant to consider the case where the ambient space is the three-dimensional Euclidean space.

In this paper we address the problem of designing control laws to stabilize motion patterns in a model of identical particles moving at unit speed in three-dimensional Euclidean space. This work builds on previous works on planar formation control laws [6], [7] extending the main results to the three-dimensional setting. First we consider the case of all-to-all communication among the agents, and we derive a (static) control law which uses only measurements about relative positions and orientations of the other agents.

All-to-all communication is an assumption that is often unrealistic in multi-agent systems. In particular, in a network of moving agents, some of the existing communication links can fail and new links can appear when other agents enter an effective range of detection. To extend the all-to-all feedback design to the situation of limited communication, we use the approach recently proposed in [8], see also [9] and [10] for related work.

This approach suggests to replace the average quantities often required in a collective optimization algorithm by local estimates provided by a consensus estimator. The idea has been successfully applied to the problem of synchronization and balancing in phase models in the limited communication case [11], [8] or to the design of planar collective motions

This paper presents research results of the Belgian Network DYSCO (Dynamical Systems, Control, and Optimization), funded by the Interuniversity Attraction Poles Programme, initiated by the Belgian State, Science Policy Office. The scientific responsibility rests with its authors.

L. Scardovi and R. Sepulchre are with the Department of Electrical Engineering and Computer Science, University of Liège, Belgium, 1.scardovi@ulg.ac.be,r.sepulchre@ulg.ac.be

N.E. Leonard is with the Department of Mechanical and Aerospace Engineering, Princeton University, Princeton, NJ 08544, USA, naomi@princeton.edu
[7]. On the bases of these results we design control laws that globally stabilize collective motion patterns under mild assumptions on the communication topology.

The rest of the paper is organized as follows: in Section II we define the kinematic model for a group of steering particles moving in three-dimensional Euclidean space with unitary velocity. In Section III we derive control laws that stabilize parallel and circular formations, the only relative equilibria of the model, in the presence of all-to-all communication among the agents. Finally, in Section IV, we generalize our all-to-all design to general communication topologies.

\section{A KINEMATIC MODEL OF STEERED PARTICLES IN $\mathrm{SE}(3)$}

We consider a kinematic model of $\mathrm{N}$ identical particles (with unitary mass) moving in three-dimensional Euclidean space at unit speed:

$$
\begin{aligned}
\dot{\boldsymbol{r}}_{k} & =\boldsymbol{x}_{k} \\
\dot{\boldsymbol{x}}_{k} & =\boldsymbol{y}_{k} u_{k}+\boldsymbol{z}_{k} v_{k} \\
\dot{\boldsymbol{y}}_{k} & =-\boldsymbol{x}_{k} u_{k} \\
\dot{\boldsymbol{z}}_{k} & =-\boldsymbol{x}_{k} v_{k} \quad k=1, \ldots, N,
\end{aligned}
$$

where $\boldsymbol{r}_{k} \in \mathbb{R}^{3}$ denotes the position of particle $k$, $\left(\boldsymbol{x}_{k}, \boldsymbol{y}_{k}, \boldsymbol{z}_{k}\right)$ is a right handed orthonormal frame associated to particle $k$ (in particular $\boldsymbol{x}_{k} \in S_{2}$ is the (unit) velocity vector). The scalars $u_{k}, v_{k}$ represent the curvature controls of the $k$ th particle. We use a bold variable without index to denote the corresponding $3 N$-dimensional vector, e.g. $\boldsymbol{x}=\left(\boldsymbol{x}_{1}^{T}, \ldots, \boldsymbol{x}_{N}^{T}\right)^{T}$.

If the curvature controls are feedback functions of shape quantities (i.e. relative frame orientations and relative positions), the closed loop vector field is invariant under the action of the symmetry group $S E(3)$. The resulting closed loop dynamics evolve in a quotient manifold called shape space and the equilibria of the reduced dynamics are called relative equilibria. Relative equilibria of the model (1) have been characterized in [3]. The equilibria are of two types: parallel motion, and circular motion in planes orthogonal to the same axis of rotation. With a slight modification of the model (1), a third type of relative equilibria exists, i.e. helical motions (see [3] for details). Recently, in [4], a new framework is proposed which allows to deal with this kind of relative equilibrium.

The purpose of this paper is to design control laws, depending only on shape quantities, which stabilize the relative equilibria of the model (1). If the feedback laws depend on relative orientations only, then the model (1) 
reduces to the model

$$
\begin{aligned}
& \dot{\boldsymbol{x}}_{k}=\boldsymbol{y}_{k} u_{k}+\boldsymbol{z}_{k} v_{k} \\
& \dot{\boldsymbol{y}}_{k}=-\boldsymbol{x}_{k} u_{k} \\
& \dot{\boldsymbol{z}}_{k}=-\boldsymbol{x}_{k} v_{k} \quad k=1, \ldots, N .
\end{aligned}
$$

This model can be conveniently rewritten in a compact form. The state

$$
R_{k}=\left[\boldsymbol{x}_{k}, \boldsymbol{y}_{k}, \boldsymbol{z}_{k}\right] \in S O(3)
$$

represents the orientation of the frame attached to particle $k$. Then (2) rewrites to

$$
\dot{R}_{k}=R_{k} U_{k}, \quad k=1, \ldots, N,
$$

where

$$
U_{k}=\left[\begin{array}{ccc}
0 & -u_{k} & -v_{k} \\
u_{k} & 0 & 0 \\
v_{k} & 0 & 0
\end{array}\right]
$$

is a skew-symmetric matrix that represents an element of the tangent space $s o(3)$.

\section{STABILIZING LAWS IN THE PRESENCE OF ALL-TO-ALL COMMUNICATION}

\section{A. Orientation stabilization}

The average linear momentum of a group of particles satisfying (1) is the centroid of the particles' velocities

$$
\overline{\boldsymbol{x}}=\frac{1}{N} \sum_{j=1}^{N} \boldsymbol{x}_{j} .
$$

The parameter $\|\overline{\boldsymbol{x}}\|$ is a measure of synchrony of the velocity vectors $\boldsymbol{x}_{k}, k=1,2, \ldots, N$. In the model (1), $\|\overline{\boldsymbol{x}}\|$ is maximal when the velocity vectors are all aligned (synchronization) leading to parallel formations. It is minimal when the velocities balance to result in a vanishing centroid, leading to collective motion around a fixed center of mass. Synchronization (balancing) is then achieved by maximizing (minimizing) the potential

$$
V(\boldsymbol{x})=\frac{N}{2}\|\overline{\boldsymbol{x}}\|^{2} .
$$

The time derivative of (4) along the solutions of (2) is

$$
\dot{V}=\sum_{j=1}^{N}<\overline{\boldsymbol{x}}, \dot{\boldsymbol{x}}_{j}>=\sum_{j=1}^{N}<\overline{\boldsymbol{x}}, \boldsymbol{y}_{j}>u_{j}+<\overline{\boldsymbol{x}}, \boldsymbol{z}_{j}>v_{j} .
$$

The control law

$$
\begin{aligned}
& u_{k}=-K<\overline{\boldsymbol{x}}, \boldsymbol{y}_{k}> \\
& v_{k}=-K<\overline{\boldsymbol{x}}, \boldsymbol{z}_{k}>, \quad k=1, \ldots, N,
\end{aligned}
$$

ensures that (4) is non-decreasing (non-increasing) when $K<0(K>0)$.

The following result provides a characterization of the equilibria of (2) under the control law (6).

Theorem 1: Consider the model (2) with the control law (6). If $K<0$ only the set of synchronized states (i.e. those states such that $\boldsymbol{x}_{k}$ are all identical) is asymptotically stable and every other equilibrium is unstable. If $K>0$ only the set of balanced states (i.e. those states such that $\overline{\boldsymbol{x}}$ is zero) is asymptotically stable and every other equilibrium is unstable.

Proof: Plugging (6) into (5) yield

$$
\dot{V}=-K \sum_{k=1}^{N}<\overline{\boldsymbol{x}}, \boldsymbol{y}_{k}>^{2}+<\overline{\boldsymbol{x}}, \boldsymbol{z}_{k}>^{2} \leq 0 .
$$

By La Salle principle, the solutions of (2) converge to the largest invariance set where

$$
\begin{aligned}
& <\overline{\boldsymbol{x}}, \boldsymbol{y}_{k}>=0 \\
& <\overline{\boldsymbol{x}}, \boldsymbol{z}_{k}>=0, \quad k=1, \ldots, N .
\end{aligned}
$$

The points where $\overline{\boldsymbol{x}}=0$, are global minima of $V$. As a consequence this equilibrium set is stable if $K>0$ and unstable if $K<0$. From (7), equilibria where $\overline{\boldsymbol{x}} \neq 0$ are characterized by the vectors $\boldsymbol{x}_{k}, k=1, \ldots, N$, all parallel to the constant vector $\overline{\boldsymbol{x}}$. Note that this configuration involves $N-M$ velocity vectors aligned to $\overline{\boldsymbol{x}}$ and $M$ velocity vectors anti-aligned to $\overline{\boldsymbol{x}}$, where $0 \leq M<\frac{N}{2}$. At those points, $\|\overline{\boldsymbol{x}}\|=1-\frac{2 M}{N}>\frac{1}{N}$. When $M=0$ we recover the set of synchronized states (global maximum of $V$ ) which is stable if $K<0$ and unstable if $K>0$. Every other value of $M$ corresponds to a saddle point and is therefore unstable both for $K>0$ and $K<0$. To see this we express $\boldsymbol{x}_{k}$ and $\overline{\boldsymbol{x}}$ in polar coordinates,

$$
\begin{aligned}
& \overline{\boldsymbol{x}}=\|\overline{\boldsymbol{x}}\|[\cos \Phi \sin \Theta, \sin \Phi \sin \Theta, \cos \Theta]^{T}, \\
& \boldsymbol{x}_{k}=\left[\cos \phi_{k} \sin \theta_{k}, \sin \phi_{k} \sin \theta_{k}, \cos \theta_{k}\right]^{T},
\end{aligned}
$$

where $\theta_{k}, \Theta \in[0, \pi]$ and $\phi_{k}, \Phi \in[0,2 \pi)$. By expressing $V$ in polar coordinates we obtain

$$
V=\|\overline{\boldsymbol{x}}\| \sum_{j=1}^{N} \sin \Theta \sin \theta_{j} \cos \left(\Phi-\phi_{j}\right)+\cos \Theta \cos \theta_{j} .
$$

The critical points are characterized by

$\boldsymbol{x}_{k}=[\cos \Phi \sin \Theta, \sin \Phi \sin \Theta, \cos \Theta]^{T}, \quad k=M+1, \ldots, N$, and

$\boldsymbol{x}_{k}=[\cos (\Phi+\pi) \sin (\pi-\Theta), \sin (\Phi+\pi) \sin (\pi-\Theta), \cos (\pi-\Theta)]^{T}$,

for $k=1, \ldots, M$.

The second derivative (with respect to $\theta_{j}$ ) is

$\frac{\partial^{2} V}{\partial \theta_{j}^{2}}=\frac{1}{N}-\|\overline{\boldsymbol{x}}\|\left(\sin \theta_{j} \sin \Theta \cos \left(\Phi-\phi_{j}\right)+\cos \Theta \cos \theta_{j}\right)$

that is negative if $\theta_{j}=\Theta$ and $\phi_{j}=\Phi$ and is positive if $\theta_{j}=\pi-\Theta$ and $\phi_{j}=\Phi+\pi$. As a consequence, a small variation $\delta \theta_{j}$ at those critical points decreases the value of $V$ if $\theta_{j}=\Theta$ and $\phi_{j}=\Phi$, and increases the value of $V$ if $\theta_{j}=\pi-\Theta$ and $\phi_{j}=\Phi+\pi$.

As a consequence of Theorem 1, we obtain that the control law (6) stabilizes parallel formations $(K<0)$ or the center of mass of the particles to a fixed point $(K>0)$ (see Fig. 1). The parallel formation is a relative equilibrium. In contrast the stabilization of the center of mass to a fixed point does not lead in general to a relative equilibrium. 

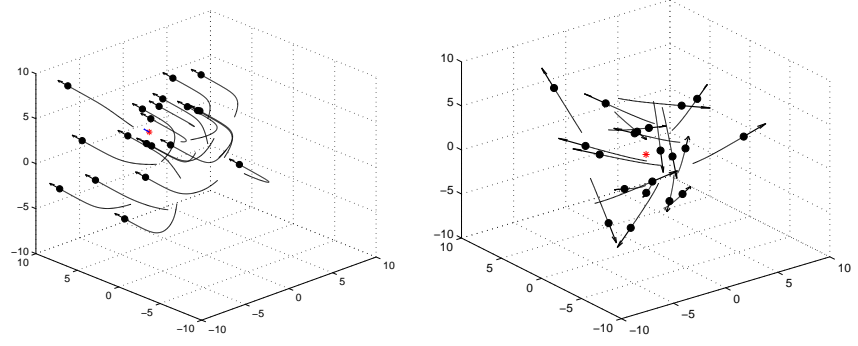

Fig. 1. Parallel and balanced formations.

Remark 1: The feedback control (6) does not depend on the relative orientation of the frames but only on the relative orientations of the velocity vectors.

\section{B. Stabilization of circular formations}

In this section we propose a spacing control to stabilize circular relative equilibria. Circular equilibria (with arbitrary relative orientations) are defined by circular motions around the same axis of rotation. Under the constant control $u_{k}=$ $\omega_{k}, v_{k}=\gamma_{k}$, with $\rho=\frac{1}{\sqrt{\omega_{k}^{2}+\gamma_{k}^{2}}}$, each particle travels a circular orbit around the (constant) axis of rotation

$$
\boldsymbol{n}_{k}=\omega_{k} \boldsymbol{z}_{k}-\gamma_{k} \boldsymbol{y}_{k},
$$

with unit speed and (common) radius $\rho$. The center of each rotating particle multiplied by the constant $\frac{1}{\rho^{2}}$ is

$$
\boldsymbol{s}_{k} \triangleq \frac{1}{\rho^{2}} \boldsymbol{r}_{k}+\boldsymbol{d}_{k} \quad k=1, \ldots N
$$

where

$$
\boldsymbol{d}_{k} \triangleq \omega_{k} \boldsymbol{y}_{k}+\gamma_{k} \boldsymbol{z}_{k} .
$$

When all centers coincide the following algebraic condition is satisfied

$$
\tilde{P} \boldsymbol{s}=0,
$$

where $P=I_{N}-\frac{1}{N} \mathbf{1 1}^{T}$ and $\tilde{P}=P \otimes I_{3}$. This suggests to define the synchrony measure

$$
S(\boldsymbol{d}, \boldsymbol{r}) \triangleq \frac{1}{2}\|\tilde{P} \boldsymbol{s}\|^{2},
$$

which is minimum when all the centers coincide. Observing that circular relative equilibria are characterized by a common axis of rotation we introduce the Lyapunov function

$$
W(\boldsymbol{n}, \boldsymbol{d}, \boldsymbol{r})=S(\boldsymbol{d}, \boldsymbol{r})-\frac{N}{2}\|\overline{\boldsymbol{n}}\|^{2}
$$

where $\overline{\boldsymbol{n}} \triangleq \frac{1}{N} \sum_{j=1}^{N} \boldsymbol{n}_{k}$. Differentiating $W$ with respect to time we obtain

$$
\begin{aligned}
\dot{W} & =\sum_{j=1}^{N}<\boldsymbol{s}_{j}-\overline{\boldsymbol{s}}, \boldsymbol{x}_{j}>\left(\frac{1}{\rho^{2}}-\omega_{j} u_{j}-\gamma_{j} v_{j}\right) \\
& -<\overline{\boldsymbol{n}}, \boldsymbol{x}_{j}>\left(\gamma_{j} u_{j}-\omega_{j} v_{j}\right) .
\end{aligned}
$$

Choosing the control

$$
\begin{aligned}
& u_{k}=\omega_{k}+<\omega_{k}\left(\boldsymbol{s}_{k}-\overline{\boldsymbol{s}}\right)+\gamma_{k} \overline{\boldsymbol{n}}, \boldsymbol{x}_{k}> \\
& v_{k}=\gamma_{k}+<\gamma_{k}\left(\boldsymbol{s}_{k}-\overline{\boldsymbol{s}}\right)-\omega_{k} \overline{\boldsymbol{n}}, \boldsymbol{x}_{k}>
\end{aligned}
$$

results in

$$
\begin{aligned}
\dot{W} & =-\sum_{j=1}^{N}<\omega_{j}\left(\boldsymbol{s}_{j}-\overline{\boldsymbol{s}}\right)+\gamma_{j} \overline{\boldsymbol{n}}, \boldsymbol{x}_{j}>^{2} \\
& +<\gamma_{j}\left(\boldsymbol{s}_{j}-\overline{\boldsymbol{s}}\right)-\omega_{j} \overline{\boldsymbol{n}}, \boldsymbol{x}_{j}>^{2} \leq 0 .
\end{aligned}
$$

Noting that

$$
\boldsymbol{s}_{k}-\overline{\boldsymbol{s}}=\frac{1}{\rho^{2}} \tilde{\boldsymbol{r}}_{k}+\boldsymbol{d}_{k}-\overline{\boldsymbol{d}}
$$

where $\tilde{\boldsymbol{r}}_{k}=\boldsymbol{r}_{k}-\overline{\boldsymbol{r}}$ and $\overline{\boldsymbol{r}}$ is the center of mass, we rewrite the control law (11) as

$$
\begin{aligned}
& u_{k}=\omega_{k}+<\omega_{k} \rho^{-2} \tilde{\boldsymbol{r}}_{k}+\gamma_{k} \overline{\boldsymbol{n}}-\omega_{k} \overline{\boldsymbol{d}}, \boldsymbol{x}_{k}> \\
& v_{k}=\gamma_{k}+<\gamma_{k} \rho^{-2} \tilde{\boldsymbol{r}}_{k}-\omega_{k} \overline{\boldsymbol{n}}-\gamma_{k} \overline{\boldsymbol{d}}, \boldsymbol{x}_{k}>,
\end{aligned}
$$

where we used the condition $\left\langle\boldsymbol{n}_{k}, \boldsymbol{x}_{k}\right\rangle=0$.

Note that $\boldsymbol{s}_{k}$ and $\boldsymbol{n}_{k}$ obey to the following dynamics

$$
\begin{aligned}
\dot{\boldsymbol{n}}_{k} & =-\boldsymbol{x}_{k}\left(\omega_{k}\left(v_{k}-\gamma_{k}\right)-\gamma_{k}\left(u_{k}-\omega_{k}\right)\right) \\
\dot{\boldsymbol{s}}_{k} & =-\boldsymbol{x}_{k}\left(\omega_{k}\left(u_{k}-\omega_{k}\right)+\gamma_{k}\left(v_{k}-\gamma_{k}\right)\right) .
\end{aligned}
$$

Plugging (12) into (13) we obtain

$$
\begin{aligned}
\dot{\boldsymbol{n}}_{k} & =-\rho^{-2} \boldsymbol{x}_{k}<\boldsymbol{n}_{k}-\overline{\boldsymbol{n}}, \boldsymbol{x}_{k}> \\
\dot{\boldsymbol{s}}_{k} & =-\rho^{-2} \boldsymbol{x}_{k}<\boldsymbol{s}_{k}-\overline{\boldsymbol{s}}, \boldsymbol{x}_{k}>.
\end{aligned}
$$

The following result characterizes the closed-loop dynamics.

Theorem 2: Consider model (13) with the feedback control (12). All the solutions converge to the equilibrium set of (13). Furthermore, every equilibrium of (13) which does not correspond to a circular relative equilibrium of (1) is unstable.

Proof: By the La Salle invariance principle we obtain that the solutions converge to the largest invariance set where

$$
\begin{gathered}
\omega_{k}<\boldsymbol{s}_{k}-\overline{\boldsymbol{s}}, \boldsymbol{x}_{k}>=-\gamma_{k}<\boldsymbol{n}_{k}-\overline{\boldsymbol{n}}, \boldsymbol{x}_{k}> \\
\gamma_{k}<\boldsymbol{s}_{k}-\overline{\boldsymbol{s}}, \boldsymbol{x}_{k}>=\omega_{k}<\boldsymbol{n}_{k}-\overline{\boldsymbol{n}}, \boldsymbol{x}_{k}>,
\end{gathered}
$$

for every $k$. In this set $u_{k}=\omega_{k}$ and $v_{k}=\gamma_{k}$ and therefore $\overline{\boldsymbol{n}}$ is constant. Moreover (15) can hold only if $\left\langle\boldsymbol{n}_{k}-\overline{\boldsymbol{n}}, \boldsymbol{x}_{k}\right\rangle=$ 0 , for every $k$ and (15) reduces to

$$
\begin{aligned}
<\boldsymbol{s}_{k}-\overline{\boldsymbol{s}}, \boldsymbol{x}_{k}> & =0 \\
<\boldsymbol{n}_{k}-\overline{\boldsymbol{n}}, \boldsymbol{x}_{k}> & =0, \quad k=1,2, \ldots, N .
\end{aligned}
$$

Conditions (16) characterize the equilibria of system (14). Only two types of equilibria are possible. The first is characterized by the conditions $\overline{\boldsymbol{n}}=0$ and $\left\langle\boldsymbol{s}_{k}-\overline{\boldsymbol{s}}, \boldsymbol{x}_{k}\right\rangle=0$. Such equilibria are unstable under the control law (12) (the analysis is analogous to the one in Theorem 1). When $\bar{n} \neq 0$ condition (16) implies that the particles rotate in planes orthogonal to the constant axis of rotation $\bar{n}$ with the same angular velocity. We conclude that $s_{k}-\bar{s}$ is parallel to $\bar{n}$ for every $k$ and that the centers lie on the same rotation axis (see Fig. 2).

Remark 2: If the open-loop control is the same for each particle, i.e. $\omega_{i}=\omega_{j}=\omega$ and $\gamma_{i}=\gamma_{j}=\gamma$ for every $i, j$, the control law (12) simplifies to

$$
\begin{aligned}
& u_{k}=\omega+\rho^{-2}<\omega \tilde{\boldsymbol{r}}_{k}-\overline{\boldsymbol{y}}, \boldsymbol{x}_{k}> \\
& v_{k}=\gamma+\rho^{-2}<\gamma \tilde{\boldsymbol{r}}_{k}-\overline{\boldsymbol{z}}, \boldsymbol{x}_{k}>
\end{aligned}
$$

where $\overline{\boldsymbol{y}} \triangleq \frac{1}{N} \sum_{j=1}^{N} \boldsymbol{y}_{k}$ and $\overline{\boldsymbol{z}} \triangleq \frac{1}{N} \sum_{j=1}^{N} \boldsymbol{z}_{k}$. 


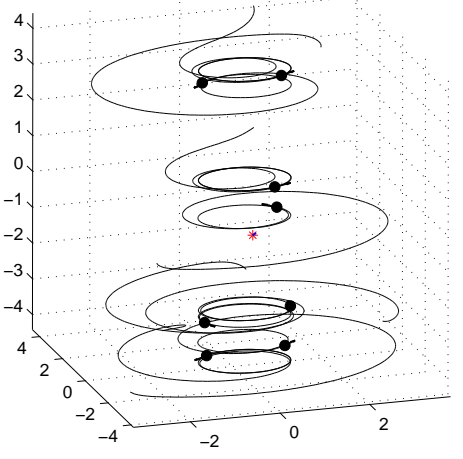

Fig. 2. Circular equilibrium achieved under the control (12).

\section{STABILIZING LAWS With LIMITED COMMUNICATION}

In this Section we extend the results of Section III when communication among the particles is limited.

\section{A. Communication graphs and consensus dynamics}

We describe the communication topology by using the notion of communication graph.

Let $G=(\mathcal{V}, \mathcal{E}, A)$ be a weighted digraph (directed graph) where $\mathcal{V}=\left\{v_{1}, \ldots, v_{N}\right\}$ is the set of nodes, $\mathcal{E} \subseteq \mathcal{V} \times \mathcal{V}$ is the set of edges, and $A$ is a weighted adjacency matrix with nonnegative elements $a_{k j}$. The node indices belong to the set of positive integers $\mathcal{I} \triangleq\{1, \ldots, N\}$. Assume that there are no self-cycles i.e. $a_{k k}=0, \forall k \in \mathcal{I}$.

The graph Laplacian $L$ associated to the graph $G$ is defined as

$$
L_{k j}= \begin{cases}\sum_{i} a_{k i}, & j=k \\ -a_{k j}, & j \neq k .\end{cases}
$$

The $k$-th row of $L$ is defined by $L_{k}$. The in-degree (respectively out-degree) of node $v_{k}$ is defined as $d_{k}^{i n}=\sum_{j=1}^{N} a_{k j}$ (respectively $d_{k}^{\text {out }}=\sum_{j=1}^{N} a_{j k}$ ). The digraph $G$ is said to be balanced if the in-degree and the out-degree of each node are equal, that is,

$$
\sum_{j} a_{k j}=\sum_{j} a_{j k}, \quad \forall k \in \mathcal{I} .
$$

It is both of theoretical and practical interest to consider time-varying communication topologies. For example, in a network of moving agents, some of the existing links can fail and new links can appear when other agents enter an effective range of detection. In the following we assume that the communication topology is described by a time-varying graph $G(t)=(\mathcal{V}, \mathcal{E}(t), A(t))$, where $A(t)$ is piece-wise continuous and bounded and $a_{k j}(t) \in\{0\} \cup[\beta, \gamma], \forall k, j$, for some finite scalars $0<\beta \leq \gamma$ and for all $t \geq 0$. The set of neighbors of node $v_{k}$ at time $t$ is denoted by $\mathcal{N}_{k}(t) \triangleq\left\{v_{j} \in \mathcal{V}: a_{k j}(t) \geq \beta\right\}$. We recall two definitions that characterize the concept of uniform connectivity for time-varying graphs.

Definition 1: Consider a graph $G(t)=(\mathcal{V}, \mathcal{E}(t), A(t))$. A node $v_{k}$ is said to be connected to node $v_{j}\left(v_{j} \neq v_{k}\right)$ in the interval $I=\left[t_{a}, t_{b}\right]$ if there is a path from $v_{k}$ to $v_{j}$ which respects the orientation of the edges for the directed graph $\left(\mathcal{N}, \cup_{t \in I} \mathcal{E}(t), \int_{I} A(\tau) d \tau\right)$.

Definition 2: $G(t)$ is said to be uniformly connected if there exists a time horizon $T>0$ and an index $k$ such that for all $t$ all the nodes $v_{j}(j \neq k)$ are connected to node $k$ across $[t, t+T]$.

Consider a group of $N$ agents with state $\boldsymbol{w}_{k} \in W$, where $W$ is an Euclidean space. The communication between the $N$-agents is defined by the graph $G$ : each agent can sense only the neighboring agents, i.e. agent $j$ receives information from agent $i$ iff $i \in \mathcal{N}_{j}(t)$.

Consider the continuous dynamics

$$
\dot{\boldsymbol{w}}_{k}=\sum_{j=1}^{N} a_{k j}(t)\left(\boldsymbol{w}_{j}-\boldsymbol{w}_{k}\right), \quad \forall k \in \mathcal{I} .
$$

Using the Laplacian definition, (18) can be equivalently expressed as

$$
\dot{w}=-\tilde{L}(t) \boldsymbol{w},
$$

where $\tilde{L}=L \otimes I_{3}$. Algorithm (19) has been widely studied in the literature and asymptotic convergence to a consensus value holds under mild assumptions on the communication topology. The following theorem summarizes some of the main results in [13], [14] and [15].

Theorem 3: Let $W$ be a finite-dimensional Euclidean space. Let $G(t)$ be a uniformly connected digraph and $L(t)$ the corresponding Laplacian matrix bounded and piecewise continuous in time. The solutions of (19) asymptotically converge to a consensus value $\alpha \mathbf{1}$ for some $\alpha \in W$. Furthermore if $G(t)$ is balanced for all $t$, then $\alpha=\frac{1}{N} \sum_{i \in \mathcal{I}} \boldsymbol{w}_{i}(0)$.

A general proof for Theorem 3 is based on the property that the convex hull of vectors $\boldsymbol{w}_{k} \in W$ is non expanding along the solutions. For this reason, the assumption that $W$ is an Euclidean space is essential (see e.g. [14]). Under the additional balancing assumption on $G(t)$, it follows that $\mathbf{1}^{T} L(t)=0$, which implies that the average $\frac{1}{N} \sum_{j \in \mathcal{I}} \boldsymbol{w}_{j}$ is an invariant quantity along the solutions.

\section{B. Orientation stabilization with limited communication}

In Section III we presented a control law that stabilizes parallel formations and balanced formations in the presence of all-to-all communication among the agents. Along the lines of [11], [8], [7] we replace the (global information) control law (6) with a local one where the quantity $\overline{\boldsymbol{x}}$ is substituted by a (local) consensus variable. Consider first the problem of synchronizing the velocity vectors $\boldsymbol{x}_{k}$. We replace the (global information) control law

$$
\begin{aligned}
& u_{k}=<\overline{\boldsymbol{x}}, \boldsymbol{y}_{k}> \\
& v_{k}=<\overline{\boldsymbol{x}}, \boldsymbol{z}_{k}>, \quad k=1, \ldots, N,
\end{aligned}
$$

with the local one

$$
\begin{aligned}
& u_{k}=<\boldsymbol{w}_{k}, \boldsymbol{y}_{k}> \\
& v_{k}=<\boldsymbol{w}_{k}, \boldsymbol{z}_{k}>, \quad k=1, \ldots, N,
\end{aligned}
$$


where $\boldsymbol{w}_{k}$ is a consensus variable obeying the consensus dynamics

$$
\dot{\boldsymbol{w}}_{k}=-\sum_{j=1}^{N} L_{k j} \boldsymbol{w}_{j}
$$

with arbitrary initial conditions $\boldsymbol{w}_{k}(0), k=1, \ldots, N$. Before detailing the convergence analysis we express (21) and (22) in shape coordinates. To this end we adopt the change of variables

$$
\boldsymbol{b}_{k}=R_{k}^{T} \boldsymbol{w}_{k}
$$

Then (21) rewrites as

$$
\begin{aligned}
& u_{k}=<\boldsymbol{b}_{k}, \boldsymbol{e}_{2}> \\
& v_{k}=<\boldsymbol{b}_{k}, \boldsymbol{e}_{3}>, \quad k=1, \ldots, N,
\end{aligned}
$$

where $\left(\boldsymbol{e}_{1}, \boldsymbol{e}_{2}, \boldsymbol{e}_{3}\right)$ is the standard orthonormal basis for $\mathbb{R}^{3}$, and (22) as

$$
\dot{\boldsymbol{b}}_{k}=U_{k}^{T} \boldsymbol{b}_{k}-\sum_{j=1}^{N} L_{k j} R_{k}^{T} R_{j} \boldsymbol{b}_{j},
$$

where $\boldsymbol{b}_{k}(0)=R_{k}^{T} \boldsymbol{w}_{k}(0)$.

Theorem 4: Let $G(t)$ be a uniformly connected communication graph and $L(t)$ the corresponding bounded and piecewise continuous Laplacian. Then all the solutions of the model (2) with the control (21) asymptotically converge to an equilibrium. Moreover, the only asymptotically stable equilibrium in the shape space is the synchronized state characterized by $N$ identical velocity vectors and every other equilibrium is unstable. Furthermore, if $G(t)$ is balanced for all $t$ and $\boldsymbol{b}_{k}(0)=\boldsymbol{e}_{1}$, for all $k \in \mathcal{I}$, then the asymptotic consensus value for $\boldsymbol{x}_{k}$ is $\boldsymbol{\alpha}=\frac{1}{N} \sum_{i \in \mathcal{I}} \boldsymbol{x}_{i}(0)$, that is the centroid $\bar{x}$ of the initial condition.

Proof: Set $\boldsymbol{w}_{k}=R_{k} \boldsymbol{b}_{k}$. Then $\boldsymbol{w}(t)$ obeys the consensus dynamics $\dot{\boldsymbol{w}}=-\tilde{L}(t) \boldsymbol{w}$, which implies that the solutions converge to a consensus value $\alpha$. Since the consensus dynamics for $\boldsymbol{w}(t)$ are invariant with respect to translations in the space, for any particular graph sequence, $\boldsymbol{\alpha}$ has an equal probability to take any value in $\mathbb{R}^{3}$ if the initial conditions $\boldsymbol{w}_{k}(0)$ are randomly chosen. This is sufficient to conclude that $\boldsymbol{\alpha} \neq 0$ with probability 1 . This implies that the control law

$$
\begin{aligned}
& u_{k}=<\boldsymbol{b}_{k}, \boldsymbol{e}_{2}> \\
& v_{k}=\left\langle\boldsymbol{b}_{k}, \boldsymbol{e}_{3}>,\right.
\end{aligned}
$$

asymptotically converges to the (time-invariant) control

$$
\begin{aligned}
& u_{k}=\left\langle\boldsymbol{\alpha}, \boldsymbol{y}_{k}>\right. \\
& v_{k}=\left\langle\boldsymbol{\alpha}, \boldsymbol{z}_{k}>,\right.
\end{aligned}
$$

for every $k \in \mathcal{I}$ and system (2) with the control (25) converges to the (time-invariant) dynamics

$$
\begin{aligned}
& \dot{\boldsymbol{x}}_{k}=\boldsymbol{y}_{k}<\boldsymbol{\alpha}, \boldsymbol{y}_{k}>+\boldsymbol{z}_{k}<\boldsymbol{\alpha}, \boldsymbol{z}_{k}> \\
& \dot{\boldsymbol{y}}_{k}=-\boldsymbol{x}_{k}<\boldsymbol{\alpha}, \boldsymbol{y}_{k}> \\
& \dot{\boldsymbol{z}}_{k}=-\boldsymbol{x}_{k}<\boldsymbol{\alpha}, \boldsymbol{z}_{k}>\quad k=1, \ldots, N .
\end{aligned}
$$

Solutions of the complete system are known to converge to a chain recurrent set of the limiting (autonomous) system
(27) [16]. The limiting system is decoupled into $N$ identical systems whose only chain recurrent sets are the equilibria of (27). In this set $\boldsymbol{y}_{k}$ and $\boldsymbol{z}_{k}$ are orthogonal to $\boldsymbol{\alpha}$ and the equilibria are two isolated points modulo the action of the symmetry group $S O(2)$ (one stable and one unstable). Then the only limit sets of the system (2) with the control (25) are equilibria characterized by $\boldsymbol{x}_{k}= \pm \frac{\boldsymbol{\alpha}}{\|\boldsymbol{\alpha}\|}$ for every $k$. The synchronized equilibrium $\boldsymbol{x}_{k}=\frac{\boldsymbol{\alpha}}{\|\boldsymbol{\alpha}\|}$ is exponentially stable while the equilibrium $\boldsymbol{x}_{k}=-\frac{\boldsymbol{\alpha}}{\|\boldsymbol{\alpha}\|}$ is unstable. If $G(t)$ is balanced, it follows from Theorem 3 that $\boldsymbol{\alpha}=$ $\frac{1}{N} \sum_{i \in \mathcal{I}} R_{i}(0) \boldsymbol{b}_{i}(0)=\overline{\boldsymbol{x}}(0)$.

Consider now the problem of balancing the velocity vectors. We replace

$$
\begin{aligned}
& u_{k}=-<\overline{\boldsymbol{x}}, \boldsymbol{y}_{k}> \\
& v_{k}=-<\overline{\boldsymbol{x}}, \boldsymbol{z}_{k}>, \quad k=1, \ldots, N,
\end{aligned}
$$

with

$$
\begin{aligned}
& u_{k}=-<\boldsymbol{w}_{k}, \boldsymbol{y}_{k}> \\
& v_{k}=-<\boldsymbol{w}_{k}, \boldsymbol{z}_{k}>, \quad k=1, \ldots, N,
\end{aligned}
$$

where $\boldsymbol{w}_{k}$ is a consensus variable obeying the consensus dynamics

$$
\dot{\boldsymbol{w}}_{k}=-\sum_{j=1}^{N} L_{k j} \boldsymbol{w}_{j}+\frac{d}{d t} \boldsymbol{x}_{k},
$$

where $\boldsymbol{w}_{k}(0)=\boldsymbol{x}_{k}(0)$ for every $k$.

In shape coordinates we obtain

$$
\begin{aligned}
& u_{k}=-<\boldsymbol{b}_{k}, \boldsymbol{e}_{2}> \\
& v_{k}=-<\boldsymbol{b}_{k}, \boldsymbol{e}_{3}>, \quad k=1, \ldots, N,
\end{aligned}
$$

where $\boldsymbol{w}_{k}$ is a consensus variable obeying the consensus dynamics

$$
\dot{\boldsymbol{b}}_{k}=U_{k}^{T}\left(\boldsymbol{b}_{k}-\boldsymbol{e}_{1}\right)-\sum_{j=1}^{N} L_{k j} R_{k}^{T} R_{j} \boldsymbol{w}_{j},
$$

where $\boldsymbol{b}_{k}(0)=\boldsymbol{e}_{1}$ for every $k$.

Theorem 5: Suppose that the communication graph $G(t)$ is uniformly connected and balanced for all $t \geq 0$ and that $L(t)$ is bounded and piecewise continuous. Then all the solutions of the system (2) with the control (29) asymptotically converge to an equilibrium. Moreover, the only stable limit set is the set of balanced states characterized by $\overline{\boldsymbol{x}}=0$.

Proof: Set $\boldsymbol{w}_{k}=R_{k} \boldsymbol{b}_{k}$. The solution $\boldsymbol{w}(t)$ satisfies the dynamics

$$
\dot{\boldsymbol{w}}=-\tilde{L}(t) \boldsymbol{w}+\frac{d}{d t} \boldsymbol{x}(t) .
$$

The Lyapunov function

$$
W(\boldsymbol{w})=\frac{1}{2}<\boldsymbol{w}, \boldsymbol{w}>,
$$


is not increasing along the solutions: note that, since the graph is balanced, $L(t)$ is a positive semi-definite matrix [17] and then

$$
\begin{aligned}
\dot{W} & =-<\tilde{L}(t) \boldsymbol{w}, \boldsymbol{w}>-\sum_{k=1}^{N}<\boldsymbol{w}_{k}, \boldsymbol{y}_{k}>^{2}+<\boldsymbol{w}_{k}, \boldsymbol{z}_{k}>^{2} \\
& =-<\tilde{L}(t) \boldsymbol{w}, \boldsymbol{w}>-\sum_{k=1}^{N}\left\|\dot{\boldsymbol{x}}_{k}\right\|^{2} \leq 0 .
\end{aligned}
$$

We deduce from (34) that $\dot{\boldsymbol{x}}$ is a function in $L_{2}(0, \infty)$. We also deduce from (34) that $\boldsymbol{w}(t)$ is uniformly bounded. To prove that $\dot{\boldsymbol{x}}$ asymptotically converges to zero observe that

$$
\ddot{\boldsymbol{x}}_{k}=-\left(u_{k}^{2}+v_{k}^{2}\right) \boldsymbol{x}_{k}
$$

is uniformly bounded (because $\boldsymbol{w}(t)$ is uniformly bounded), which implies that $\dot{\boldsymbol{x}}$ is Lipschitz continuous. We conclude that $\dot{\boldsymbol{x}}$ is uniformly continuous. Then $\dot{\boldsymbol{x}}$ is a uniformly continuous function in $L_{2}(0, \infty)$ and from Barbalat's Lemma we obtain that $\dot{\boldsymbol{x}} \rightarrow 0$ as $t \rightarrow \infty$ [18].

Thanks to the balancing assumption on the graph, $\mathbf{1}$ is a left eigenvector of $L(t)$, and we obtain from (33) that

$$
\frac{1}{N} \sum_{k=1}^{N} \dot{\boldsymbol{w}}_{k}=\frac{1}{N} \sum_{k=1}^{N} \frac{d}{d t} \boldsymbol{x}_{k} .
$$

Integrating both sides of (35), and using the fact that $\boldsymbol{w}_{k}(0)=\boldsymbol{x}_{k}(0)$, one concludes that $\frac{1}{N} \sum_{i \in \mathcal{I}} \boldsymbol{w}_{i}(t)=\overline{\boldsymbol{x}}$ for all $t \geq 0$. Because $\boldsymbol{w}(t)$ converges to a consensus equilibrium, each component $\boldsymbol{w}_{k}$ must asymptotically converge to $\overline{\boldsymbol{x}}$. As a consequence, the control law (31) asymptotically converge to the time-invariant control

$$
\begin{aligned}
& u_{k}=-<\overline{\boldsymbol{x}}, \boldsymbol{y}_{k}> \\
& v_{k}=-<\overline{\boldsymbol{x}}, \boldsymbol{z}_{k}>, \quad k=1, \ldots, N .
\end{aligned}
$$

Since $\dot{x}$ is asymptotically convergent to zero, the solutions asymptotically converge to a set of equilibria of (2) with the control law (36). We conclude that $\boldsymbol{x}(t)$ asymptotically converges to the critical set of $V$ and, form Theorem 1, that only the set of balanced states is asymptotically stable.

\section{Stabilization of circular equilibria with limited commu- nication}

In this Section we extend the results of Section III-B to the limited communication case. The (all-to-all) control law derived in Section III-B is

$$
\begin{aligned}
& u_{k}=\omega_{k}+<\omega_{k} \rho^{-2} \tilde{\boldsymbol{r}}_{k}+\gamma_{k} \overline{\boldsymbol{n}}-\omega_{k} \overline{\boldsymbol{d}}, \boldsymbol{x}_{k}> \\
& v_{k}=\gamma_{k}+<\gamma_{k} \rho^{-2} \tilde{\boldsymbol{r}}_{k}-\omega_{k} \overline{\boldsymbol{n}}-\gamma_{k} \overline{\boldsymbol{d}}, \boldsymbol{x}_{k}>.
\end{aligned}
$$

Along the lines of the previous section we substitute (37) with the (local) control laws

$$
\begin{aligned}
& u_{k}=\omega_{k}+<-\omega_{k} \rho^{-2} \boldsymbol{a}_{k}+\gamma_{k} \boldsymbol{b}_{k}-\omega_{k} \boldsymbol{c}_{k}, \boldsymbol{e}_{1}> \\
& v_{k}=\gamma_{k}+<-\gamma_{k} \rho^{-2} \boldsymbol{a}_{k}-\omega_{k} \boldsymbol{b}_{k}-\gamma_{k} \boldsymbol{c}_{k}, \boldsymbol{e}_{1}>
\end{aligned}
$$

where the $N$-dimensional consensus variables $\boldsymbol{a}_{k}, \boldsymbol{b}_{k}$ and $\boldsymbol{c}_{k}$ are computed according to the dynamics (directly computed in shape coordinates)

$$
\begin{aligned}
\dot{\boldsymbol{a}}_{k} & =U_{k}^{T} \boldsymbol{a}_{k}-\boldsymbol{e}_{1}-\sum_{j=1}^{N} L_{k j} R_{k}^{T} R_{j} \boldsymbol{a}_{j}-\sum_{j=1}^{N} L_{k j} R_{k}^{T} \boldsymbol{r}_{j}, \\
\dot{\boldsymbol{b}}_{k} & =U_{k}^{T} \boldsymbol{b}_{k}-\sum_{j=1}^{N} L_{k j} R_{k}^{T} R_{j} \boldsymbol{b}_{j} \\
\dot{\boldsymbol{c}}_{k} & =U_{k}^{T} \boldsymbol{c}_{k}-\sum_{j=1}^{N} L_{k j} R_{k}^{T} R_{j} \boldsymbol{c}_{j} .
\end{aligned}
$$

Theorem 6: Let $G(t)$ be a uniformly connected communication graph and $L(t)$ the corresponding bounded and piecewise continuous Laplacian. Then the only stable limit set of (1) equipped with the control (38) is the set of relative equilibria defined by circular orbits with the same radius around the same axis of rotation.

Proof: Set $\boldsymbol{w}_{k}=R_{k} \boldsymbol{a}_{k}+\boldsymbol{r}_{k}, \boldsymbol{q}_{k}=R_{k} \boldsymbol{b}_{k}$ and $\boldsymbol{p}_{k}=R_{\tilde{\tilde{L}}} \boldsymbol{c}_{k}$. These variables obey to the consensus dynamics $\dot{\boldsymbol{w}}=-\tilde{L} \boldsymbol{w}$, $\dot{\boldsymbol{q}}=-\tilde{L} \boldsymbol{q}$ and $\dot{\boldsymbol{p}}=-\tilde{L} \boldsymbol{p}$ respectively and the solutions converge to the consensus values $\overline{\boldsymbol{w}}, \overline{\boldsymbol{q}}$ and $\overline{\boldsymbol{p}}$. We obtain that (38) converge to the time-invariant control laws

$$
\begin{aligned}
& u_{k}=\omega_{k}+<\omega_{k} \rho^{-2}\left(\boldsymbol{r}_{k}-\overline{\boldsymbol{w}}\right)+\gamma_{k} \overline{\boldsymbol{q}}-\omega_{k} \overline{\boldsymbol{p}}, \boldsymbol{x}_{k}> \\
& v_{k}=\gamma_{k}+<\gamma_{k} \rho^{-2}\left(\boldsymbol{r}_{k}-\overline{\boldsymbol{w}}\right)-\omega_{k} \overline{\boldsymbol{q}}-\gamma_{k} \overline{\boldsymbol{p}}, \boldsymbol{x}_{k}>
\end{aligned}
$$

The potential

$$
W_{k}=\left\|\rho^{-2}\left(\boldsymbol{r}_{k}-\overline{\boldsymbol{w}}\right)-\omega_{k} \overline{\boldsymbol{p}}\right\|^{2}+\left\|\boldsymbol{n}_{k}-\overline{\boldsymbol{q}}\right\|^{2}
$$

satisfies

$$
\begin{aligned}
\dot{W}_{k}= & -<\omega_{k} \rho^{-2}\left(\boldsymbol{r}_{k}-\overline{\boldsymbol{w}}\right)-\omega_{k} \overline{\boldsymbol{p}}+\gamma_{k} \overline{\boldsymbol{q}}, \boldsymbol{x}_{k}>^{2} \\
& -<\gamma_{k} \rho^{-2}\left(\boldsymbol{r}_{k}-\overline{\boldsymbol{w}}\right)-\gamma_{k} \overline{\boldsymbol{p}}-\omega_{k} \overline{\boldsymbol{q}}, \boldsymbol{x}_{k}>^{2} \leq 0,
\end{aligned}
$$

along the solutions of (39). Following the same lines of the proof of Theorem 4 we conclude that the only stable limit set is the set of relative equilibria characterized by circular orbits with the same radius around the same axis of rotation.

\section{CONCLUSION}

In this paper we proposed control laws that stabilize parallel and circular relative equilibria in a model of identical, steered particles moving in three-dimensional Euclidean space. In the presence of general communication topologies we recovered the asymptotic convergence to relative equilibria by equipping each agent with a consensus estimator. An open question, that is a motivation for further research, is the derivation of shape control laws to stabilize helical relative equilibria. Recently, in [4], a methodology has been proposed that allows to stabilize also this type of relative equilibrium.

\section{REFERENCES}

[1] N. Leonard, D. Paley, F. Lekien, R. Sepulchre, D. Fratantoni, and R. Davis, "Collective motion, sensor networs and ocean sampling," in Proceedings of the IEEE, vol. 95, no. 1, pp. 48-74, 2007.

[2] J. Cortes, S. Martinez, T. Karatas, and F. Bullo, "Coverage Control for Mobile Sensing Networks," IEEE Trans. on Robotics and Automation, vol. 20, no. 2, pp. 243-255, 2004.

[3] E. W. Justh and P. S. Krishnaprasad, "Natural frames and interacting particles in three dimensions," in Proceedings of the 44th IEEE Conference on Decision and Control and European Control Conference, Seville, Spain, 2005, pp. 2841-2846.

[4] L. Scardovi, N. Leonard, and R. Sepulchre, "Stabilization of Collective Motion in Three Dimensions: A Consensus Approach," in 46th IEEE Conference on Decision and Control, (New Orleans, LA), 2007, submitted

[5] J. Fax, R. Murray, "Information Flow and Cooperative Control of Vehicle Formations," IEEE Trans. on Automatic Control, vol. 49, no. 9, pp. 1465-1476, 2004.

[6] R. Sepulchre, D. Paley, and N. Leonard, "Stabilization of planar collective motion with all-to-all communication," accepted for publication in IEEE Trans. on Automatic Control. 
[7] - "Stabilization of planar collective motion with limited communication," IEEE Trans. on Automatic Control, 2006, conditionally accepted.

[8] L. Scardovi and R. Sepulchre, "Collective optimization over average quantities," in Proceedings of the 45th IEEE Conference on Decision and Control, San Diego, Ca, pp. 3369-3374, 2006.

[9] R. A. Freeman, P. Yang, and K. M. Lynch, "Distributed estimation and control of swarm formation statistics," in Proceedings of the American Control Conference, 2006

[10] R. Olfati-Saber, "Distributed Kalman Filter with embedded consensus filters," in Proceedings of the 44th IEEE Conference on Decision and Control, Seville, Spain, pp. 8179-8184, 2005.

[11] L. Scardovi, A. Sarlette, and R. Sepulchre, "Synchronization and Balancing on the $N$-Torus," Systems and Control Letters, vol. 56, no. 5, pp. 335-341, 2007.

[12] V. D. Blondel, J. M. Hendrickx, A. Olshevsky, and J. N. Tsitsiklis, "Convergence in multiagent coordination, consensus, and flocking," in Proceedings of the 44th IEEE Conference on Decision and Control and European Control Conference, Seville, Spain, 2005, pp. 2996-3000.

[13] L. Moreau, "Stability of continuous-time distributed consensus algorithms," in Proceedings of the 43rd IEEE Conference on Decision and Control, Atlantis, Paradise Island, Bahamas, 2004, pp. 3998-4003.

[14] _ "Stability of multi-agent systems with time-dependent communication links," IEEE Trans. on Automatic Control, vol. 50, pp. 169-182, 2005.

[15] R. Olfati-Saber and R. Murray, "Consensus problems in networks of agents with switching topology and time-delays," IEEE Trans. on Automatic Control, vol. 49, pp. 1520-1533, 2004.

[16] K. Mischaikow, H. Smith, and H. Thieme, "Asymptotically autonomous semiflows; chain recurrence and Lyapunov functions," Trans. of the American Mathematical Society, vol. 347, pp. 1669-1685, 1995.

[17] J. C. Willems, "Lyapunov functions for diagonally dominant systems," Automatica J. IFAC, vol. 12, pp. 519-523, 1976.

[18] H. K. Khalil, Nonlinear Systems. Prentice Hall, 2001. 